\title{
RIESGOS PSICOSOCIALES: NUEVOS ESCENARIOS DE ORGANIZACIÓN DEL TRABAJO Y SU EFECTO EN LA SALUD DE LOS TRABAJADORES DE UNA EMPRESA CHILENA DEL ÁREA PORTUARIA
}

\author{
PSYCHOSOCIAL RISKS: NEW SCENARIOS OF LABOR ORGANIZATION AND ITS \\ EFFECT ON THE HEALTH OF DOCKWORKERS AT A PORT IN CHILE
}

\section{Willybaldo Saavedra P. ${ }^{1}$ y Camilo Campos R. ${ }^{2}$}

\begin{abstract}
Resumen
La actividad portuaria se desarrolla dentro de un contexto donde la salud de los trabajadores se ve constantemente expuesta, dadas las múltiples situaciones peligrosas que conlleva esta actividad. Es en este contexto que las altas presiones ejercidas por la organización, traducidas en la constante presión por movilizar el mayor número de carga y cumplir con rendimientos de productividad, sumado a las relaciones interpersonales, el contenido del trabajo y la realización de la tarea, entre otras, pueden afectar tanto al desarrollo del trabajo como a la salud del trabajador, lo cual se conoce como riesgos psicosociales. El objetivo de esta investigación fue determinar el nivel de riesgo psicosocial de los trabajadores de diferentes áreas de un puerto del norte de Chile. Se diseñó un estudio descriptivo, de corte transversal, sobre 298 trabajadores, seleccionados mediante un muestreo no probabilístico. Se aplicó el instrumento SUSESO ISTAS-21, versión corta de la superintendencia de seguridad social del gobierno de Chile. En cuanto a las secciones en las que los trabajadores desempeñan sus funciones, el porcentaje mayor correspondió a personal operativo con un 45,3\% (135), y el menor fue AG. Con un 9,1\% (27). Se encontraron diferencias estadísticamente significativas entre las distintas áreas en las dimensiones exigencias psicológicas, trabajo activo y desarrollo de habilidades y compensación. Para futuras investigaciones e intervenciones organizacionales se sugiere la incorporación de metodologías de trabajo cualitativas. Se concluye que el personal portuario presenta una elevada exposición de riesgos psicosociales en el ambiente laboral.
\end{abstract}

\section{Palabras clave:}

riesgos psicosociales, salud del trabajo, modernización portuaria, trabajadores portuarios, globalización.

\begin{abstract}
The port activity is developed within a context where the health of the workers is constantly exposed, giving the multiple dangerous situations that this activity entails. Is in this context that the high demands that the organization requires traduced in the constant pressure to mobilize the most amount of load and achieve with the productivity requirements, added to the interpersonal relations, the workload, the realization of the task, among others; might affect the development of the task, like, as well, the health of the worker, this is known like psychosocial risk. The objective of this research is to determine the level of psychosocial risks at the workplace of members in different areas at a port in the north of Chile. A descriptive and cross-sectional study was carried out on 298 workers, who were selected through a non-probability based sampling. An abridged version of the SUSESO ISTAS -21 (instrument provided by the government of Chile), was applied. Regarding the sections in which workers perform their duties, the highest percentage corresponded to operational staff with a $45.3 \%$ (135) and the lowest to management and administration with a 9.1\% (27). Significant statistical differences between the areas were found in the dimensions of psychological requirements, active work \& skill development, and compensation. For future research and interventions, it is recommended to incorporate qualitative work methodologies to do a more detailed analysis. Data suggests that port workers have high exposure to psychosocial risks in their workplace.
\end{abstract}

\section{Keywords:}

psychosocial risks, worker health, port modernization, dockworkers, globalization.

Fecha de recepción: 04/11/2016.

Fecha de aceptación: 22/11/2016.

Ingeniero Civil Industrial, Licenciado en ciencias de la Ingeniería, Profesor Asistente en Fonoaudiología, Facultad de Ciencias de la Salud de la Universidad Arturo Prat, Iquique, Chile, teléfono: 56976210706, email: willy.saavedra@gmail.com

2 Licenciado en Trabajo Social, Facultad de Ciencias Jurídicas y Políticas de la Universidad Arturo Prat. Iquique, Chile, teléfono: 56984663458, email: camilo.campos@alumnos.unap.cl 


\section{INTRODUCCIÓN}

El mundo del trabajo es un fenómeno dinámico y versátil, que transforma y compromete al que lo realiza, situándolo en un contexto particular y en las limitaciones inmediatas de la situación (Teiger, 1992). El trabajo es una actividad fundamental en la vida de las personas, principalmente por la necesidad que tiene el hombre de adquirir recursos económicos que faciliten su desarrollo social y su propia supervivencia (Charria, Victor, Sarsosa, Kewi y Arenas, 2011). Sin embargo, el trabajo puede traer consigo elementos negativos para los individuos, como la insatisfacción, el disconfort, y producir además alteraciones de salud físicas y psicológicas (aislamiento, malestar, desagrado, accidentes, enfermedades o incluso muerte) (Ceballos, Valenzuela y Paravic, 2014; Astudillo, 2014), principalmente por el constante cambio al cual está sujeto el mundo del trabajo, donde el imperativo desarrollo, la constante transformación de tecnologías, la intensificación de la competencia a nivel mundial, la mayor orientación en función de los mercados, y una función más reducida del Estado (Gil Monte, 2012) propician escenarios desfavorables para los individuos.

Los sistemas de trabajo y de explotación han evolucionado desde la revolución industrial, asumiendo para el cambio, progresivamente, la organización científica (Bourdieu, 2000; Useche, 2002). Hoy en día los sistemas de trabajo son influenciados por coyunturas sociales y políticas, como la globalización de la economía, la internalización de los mercados, la competitividad y la constante innovación tecnológica (Charria et al., 2011; Peiró, 2004). Lo anterior se hace necesario para que las organizaciones puedan generar un potencial aumento de la productividad, transfigurando la forma de entender el trabajo y posicionándose en el mercado laboral contemporáneo (Barattini, 2009; Bernardo, 2009).

La globalización ha contribuido a la generación de cambios en las estructuras de trabajo a nivel mundial, teniendo su materialización principalmente en el ámbito económico, mientras que la transnacionalización de las empresas ha propiciado un escenario laboral más competitivo para los trabajadores, sometiéndolos a fuertes presiones a fin de incrementar la productividad y disminuir los costos (Garrido et al., 2011; Antunes R y Alves, 2014).

A causa de la constante presión que tienen las organizaciones por ser más competitivas y productivas, los sistemas de trabajo forjan altas exigencias a los trabajadores, focalizándose en un mayor esfuerzo en el trabajo mental por sobre el físico, el "adelgazamiento" y flexibilización de las estructuras organizacionales, la tercerización de servicios y la utilización de entornos virtuales de trabajo son unas de las tantas estrategias utilizadas por las nuevas organizaciones para sobrevivir, ser sostenibles y continuar desarrollándose en el mundo contemporáneo (Peiró, 2004; Charria et al., 2011).

Dentro de un país, el Estado es el encargado de legitimar y determinar el accionar de sus políticas. En la actualidad y bajo la coyuntura socioeconómica, el Estado neoliberal consolida su carácter desregulador, retirándose de las políticas sociales y des-responsabilizándose de las contradicciones sociales generadas por el capitalismo y su exacerbada concentración de riqueza en manos de unos pocos (Torres, 2007), beneficiando solo a grandes corporaciones que producen mayores ganancias, mientras se incrementa la pobreza y el desempleo en el mundo (Feo, 2003).

Una de las políticas sociales conflictivas se refiere al derecho del trabajador y a la condición de vida de éstos. Esta situación tiene un gran efecto sobre las condiciones laborales y la salud de los trabajadores. Las actuales relaciones de trabajo se transforman con la llegada de la globalización, ya que los trabajadores son presionados a resultados eficaces en un menor tiempo de producción, existiendo un proceso precario de trabajo en el contexto de inseguridad social para la clase trabajadora.

Los nuevos métodos de producción y de organización impactan sobre los procesos de trabajo que atingen a las más diversas actividades económicas. Una de las más importantes dentro de las nuevas actividades post globalización, por su papel como interceptor y remitente de mercancías desde y hacia naciones, son los puertos.

Los puertos tienen un rol fundamental en el capitalismo global, ya que son territorios donde se realizan las distribuciones de mercancías entre países o regiones de un mismo país; además se establecen relaciones conjuntas de trabajadores, empleadores u organismos del Estado, coexistiendo diferentes formas de trabajo (Garrido et al., 2011) y posicionando a esta actividad como foco de distribución económica.

Durante las últimas décadas, principalmente después de 1980, los puertos pasan a intensificar el trabajo y a movilizar un mayor volumen de carga contenedorizada. Las transformaciones del puerto están relacionadas con los avances tecnológicos introducidos por la 
política neoliberal con una consecuencia en la reducción de la fuerza de trabajo y una mayor intensificación de éste, impactando en la vida de los trabajadores de un puerto, principalmente en relación a las condiciónes de trabajo y bienestar (Ferreira, Lara y Mariano, 2015).

Si bien el trabajo varía considerablemente por la inserción de tecnología, las relaciones de producción por el mundo revelan considerables persistencias de las formas de organización del trabajo entre los puertos (Ferreira et al., 2015). La lógica de trabajo utilizada en los puertos es que a mayor movimientos de carga a través del uso de máquinas, menor es la cantidad de trabajo humano, por lo que el servicio se abarata (Torres, 2007).

El aumento de los movimientos de carga se correlaciona directamente con una mayor intensificación del trabajo. Rosso define la intensificación del trabajo como la derivación de los procesos de cualquier naturaleza, que resulta en una mayor utilización de las capacidades físicas, cognitivas y emocionales del trabajador, con el objetivo de aumentar cuantitativamente la producción o mejorar los resultados, en síntesis: más trabajo (Costa, 2010).

Los nuevos cambios en el trabajo portuario han ocasionado reducciones en los puestos de empleo, la extinción de funciones y la introducción de trabajadores multifuncionales, el aumento de la jornada y del ritmo de trabajo, así como el establecimiento de indicadores de productividad superiores, exigidos por los compradores de servicios (Maciel, Gonçalves, Matos, Fontenelle y Santos, 2015). El trabajo que se desarrolla en los puertos sufre alteraciones a raíz de varios aspectos: las formas de organización del trabajo, la instalación del trabajo continuo (24 horas), la reducción del tiempo de atraque de los navíos en el muelle y las transformaciones en las condiciones de trabajo, que condicionan ciertos factores que son determinantes en el desarrollo de enfermedades (Ferreira et al., 2015).

Investigaciones efectuadas en Brasil señalan que la reducción de tamaño de los equipos de trabajo y las altas presiones para producir más en menos tiempo son factores que contribuyen al deterioro de las relaciones laborales y exponen a los trabajadores a riesgos (Maciel et al., 2015). Frente a los factores determinantes de agravio de la salud, la enfermedad incide sobre los trabajadores en forma de fatigas profundas y crónicas, estrés patológico, disturbios músculo-esqueléticos, accidentes del trabajo, trastornos mentales y burnout, o más comúnmente conocido como riesgos psicosociales (Asociación Chilena de Seguridad [ACHS], 2014).
Paralelo a la intensificación del trabajo portuario, los riesgos de carácter psicosocial se han visibilizado de manera de exponer una inquietud que solo se aborda desde el mejoramiento en los niveles de producción de las organizaciones, determinados por modelos de desarrollo laboral donde prima el trabajo individualizado y sectorizado; estructuras organizacionales horizontales y rígidas; cambios en métodos de empleos como las subcontrataciones; cargos y funciones temporales y nuevas pautas en horarios laborales (Garrido et al., 2011), confluyendo en factores riesgosos para la salud física y psicológica de los trabajadores expuestos a organizaciones bajo lógica capitalista y mercantil (Antunes y Alves, 2004). Estos factores son definidos por la Organización Internacional del Trabajo y como: "Las interacciones entre el contenido, la organización y la gestión del trabajo y las condiciones ambientales, por un lado, y las funciones y necesidades de los trabajadores, por otro. Estas interacciones podrían ejercer una influencia nociva en la salud de los trabajadores a través de sus percepciones y experiencia" (Organización Internacional del Trabajo [OIT], 1986).

Por otro lado, la Agencia Europea de Salud y Seguridad en el Trabajo (EU-OSHA), determina qué factor de riesgos de características psicosociales se entiende como aspectos de la concepción, organización y gestión del trabajo, así como de su contexto social y ambiental que tienen la potencialidad de causar daños físicos, sociales o psicológicos en los trabajadores (Cox, Griffiths y Rial-González, 2000). Más aún, el cuestionario de riesgos psicosociales de el trabajo SUSESO - ISTAS 21 define riesgos psicosociales como todas las características de la organización del trabajo que puedan tener algún efecto sobre la salud en los trabajadores, estandarizando y unificando el concepto el cual en que se utiliza en Chile (SUCESO, 2009).

Numerosos artículos e investigaciones confluyen que evaluar los riesgos psicosociales y su implicancia en el medio que se desenvuelve el trabajador será determinante para el desarrollo del mismo, dado el impacto que posee en la salud tanto física como psicológica del trabajador, en su satisfacción y en su calidad de vida (Beltrán, González y Salas, 2013).

A pesar de las muchas definiciones de riesgos psicosociales, Cuenca (1996) vislumbra elementos transversales en cada una de ellas, elementos que integran por sí solos los riesgos de índole psicosocial. Cuenca determina dos conceptos. En primera instancia lo asociado a condiciones de trabajo, donde se desprenden factores como las necesidades del trabajador, las capacidades 
del mismo y las condiciones que en el contexto laboral el trabajador posea. El segundo concepto se asocia a las consecuencias, vistas desde dos parámetros diametralmente opuestos: Potencial dañino para el desarrollo del trabajo, referido a la productividad, y potencial dañino para la salud del trabajador, tanto físico como psicológico (Cuenca, 1996).

En las empresas chilenas existe desconocimiento sobre cuáles son los factores que generan actualmente más problemáticas de tipo psicosocial en el trabajo. Sin embargo, ya existen organizaciones que se han dedicado a estudiar el fenómeno en Chile y hay cierto consenso sobre cuáles serían los factores psicosociales que se encuentran con mayor frecuencia en las organizaciones, por lo que el Ministerio de Salud ha generado un protocolo de vigilancia, que busca conocer cómo se están viendo afectadas las organizaciones chilenas (Ministerio de Salud [MINSAL], 2013), además de comenzar a generar información estadística sobre este fenómeno para proveer información para tomar acciones que beneficien la salud pública del país.

En Chile es muy poco lo que se sabe del trabajo portuario y su relación con los riesgos psicosociales. Hay una falta de investigaciones que aborden la temática sobre los trabajadores portuarios, no solamente en Chile, sino que también en Latinoamérica y en el resto del mundo.

El presente estudio tuvo como objetivo determinar el nivel de riesgos psicosociales en el empleo de los trabajadores de una empresa portuaria del Norte de Chile. Para ello, en primer lugar se presentó una breve perspectiva histórica sobre la concepción del sistema de trabajo portuario. Posteriormente se analizaron los cambios recientes en el entorno social y económico y sus implicaciones sobre el sistema de trabajo portuario. En tercer lugar se buscó comprobar si existen diferencias estadísticamente significativas en las dimensiones del instrumento entre los distintos grupos. En cuarto lugar, comprobar si existen diferencias estadísticamente significativas en las sub-dimensiones del instrumento entre los distintos grupos.

\section{MÉTOdo \\ DISEÑO Y MUESTRA}

El estudio es de tipo descriptivo, con un diseño no experimental, transversal, en el que se aplicó un cuestionario autoadministrado, guardando el anonimato de los participantes, durante el primer trimestre del año 2016. La muestra es de tipo no probabilística y por conveniencia, donde se seleccionaron los trabajadores que tuvieran 6 meses o más de antigüedad en el puesto, dando un universo de 315 trabajadores Aplicando los criterios de exclusión, la muestra se compuso por 298 trabajadores de una empresa del sector portuario de la primera región, separados en cinco principales áreas: personal operativo (PO), operadores de equipo y mantenimiento (OEM), personal administrativo operativo (AO), línea de mando y supervisión (LMS), y administración y gerencia (AG).

\section{Instrumento de recolección de datos}

Se empleó el instrumento SUSESO-ISTAS 21, versión corta, adaptado y validado para la población chilena por la Universidad de Chile, la Superintendencia de Seguridad Social (SUSESO), la Dirección del Trabajo y el Instituto de Salud Pública. Este cuestionario fue diseñado como una herramienta de medición, prevención, y vigilancia epidemiológica en riesgo psicosociales e investigaciones en salud ocupacional, por lo que su validación y adaptación al contexto nacional asoma como el principal insumo para dar cuenta del daño físico, psíquico y social al que los trabajadores están expuestos. También se buscó realizar una profundización en la temática desde un punto de vista crítico a través de una metodología cuantitativa y por último generar conclusiones que propicien un mejor entendimiento del fenómeno laboral. Este instrumento permite la evaluación de riesgos psicosociales en el trabajo y consta de 5 dimensiones y 20 sub-dimensiones, en cuyo cuestionario se incluye una pregunta por cada sub-dimensión, con cinco opciones de respuesta tipo Likert, que van de 0 (nunca) a 4 (siempre), donde una mayor puntuación indica un mayor riesgo. Para su evaluación se suman los puntajes de cada apartado y se 
clasifican en tres categorías: bajo (nivel de exposición más favorable para la salud), medio (nivel intermedio) y alto (nivel de exposición más desfavorable para la salud) (Superintendencia de Salud y Seguridad Social [SUSESO], 2016). Si bien se tiene información respecto del instrumento aplicado en Chile, teniendo exigencias psicológicas con un alfa de Cronbach de 0,857, trabajo activo y desarrollo de habilidades de 0,859, apoyo social en la empresa y calidad de liderazgo de 0,915, compensación de 0,829 y doble presencia de 0,644, esto corresponde a la versión extensa. Por otro lado, la versión breve no posee información psicométrica respecto del instrumento en Chile, es por esta razón que se hizo el análisis de la confiabilidad del instrumento en esta aplicación obteniendo un alfa de Cronbach de 0,667 , el que se encuentra dentro de los límites aceptables (Alvarado, Pérez-Franco, Saavedra, Fuentealba, Alarcón, Marchetti y Aranda, 2012).

\section{Variables de estudios}

Se definieron variables referidas a características socio-demográficas y laborales de los participantes (edad, sexo, nivel de escolaridad, estado civil y antigüedad laboral) y 5 variables relativas a las 5 dimensiones, las que se definen a continuación.

Exigencias psicológicas: Esta dimensión hace referencia a dos componentes, las exigencias de tipo cuantitativo y las emocionales. Las primeras hacen referencia a los cambios en el mundo del trabajo, que en las últimas décadas han repercutido en las condiciones de trabajo y en los riesgos laborales a los que están expuestos los trabajadores, en particular en el sector portuario, donde las características de las actividades generan condiciones favorables para la aparición de riesgos psicosociales, tiempo efectivo en la realización de trabajos o tareas, cumplimiento de turnos, cantidad de carga movilizada y aplicaciones de protocolos, entre otros resultados cuantificables.

Por otro lado, las exigencias psicológicas de tipo emocionales se relacionan con las exigencias cognitivas, asociadas a la emocionalidad de los trabajadores, ámbito no profundizado en esta materia (Dormann \& Zapf, 2004; Vanroelen, Levecque, Moors, Gadeyne y Louckx, 2009).
Trabajo activo y desarrollo de habilidades: Dimensión vinculada a la integración en la empresa, en contextos de trabajos como la influencia y control en tiempos productivos; posibilidad de crecimiento y desarrollo en la actividad, ya sea en capacitaciones o en un nivel de pertenencia con las funciones y el trabajo realizado (Mursula, Vathera, Linna, Pentti y Kivimäki, 2005).

Apoyo social en la empresa y claridad del liderazgo: Destaca el apoyo social de la empresa bajo la idea funcional, de relaciones con pares y superiores, focalizando esta idea en la claridad y conflictos en materia de roles, además de calidad en liderazgo, relaciones con jefaturas y calidad de relaciones con compañeros de trabajo, ya sea en turnos y contra-turnos (Stoetzer, Ahlberg, Johansson, Bergman, Hallsten, Forsell y Lundberg, 2009).

Compensaciones: Directamente ahonda en la percepción de estima en el ámbito laboral, concretamente en reconocimientos por labores, apoyo en el cumplimiento de funciones, buen trato y recompensas. A la vez, esta dimensión encara inseguridades del trabajador en materia de contrato de trabajo y características del mismo (por ejemplo, continuidad, definición y descriptor de cargos y funciones ordenados por superiores). Esta dimensión se construye bajo el modelo esfuerzo-recompensa, y parte del supuesto de que la relación entre un esfuerzo elevado y un bajo nivel de recompensas a largo plazo representa un riesgo para la salud (Siegrit, 1996).

Doble presencia: Evalúa la existencia de dos o más exigencias en materia de responsabilidades por parte del trabajador, donde focaliza el trabajo en tareas domésticas, de crianza y familiares, considerando que responder de buena manera, tanto en el contexto laboral como en el familiar, puede complejizar y afectar de manera negativa el accionar del trabajador en sus distintos contextos (Grau, Flichtentrei, Suñer, Prats \& Braga, 2009). La realización de labores en los contextos mencionados indudablemente aumenta la carga de responsabilidades y en consecuencia plantea generar conflicto en el tiempo del trabajador, puesto que estas actividades comparten la misma realidad social. 
Tabla 1:

Composición del cuestionario SUSESO-ISTAS 21 (versión breve).

\begin{tabular}{|c|c|c|}
\hline Dimensión & Sub-dimensión & Preguntas \\
\hline \multirow{5}{*}{ Exigencias psicológicas } & $\begin{array}{l}\text { Exigencias psicológicas } \\
\text { cuantitativas }\end{array}$ & ¿Puede hacer su trabajo con tranquilidad y tenerlo al día? \\
\hline & $\begin{array}{c}\text { Exigencias psicológicas } \\
\text { cognitivas } \\
\end{array}$ & En su trabajo, ¿tiene usted que tomar decisiones difíciles? \\
\hline & $\begin{array}{c}\text { Exigencias psicológicas emocio- } \\
\text { nales }\end{array}$ & $\begin{array}{c}\text { En general, ¿considera usted que su trabajo le produce } \\
\text { desgaste emocional? }\end{array}$ \\
\hline & $\begin{array}{c}\text { Exigencias psicológicas de es- } \\
\text { conder emociones }\end{array}$ & $\begin{array}{l}\text { En su trabajo tiene usted que guardar sus emociones y no } \\
\text { expresarlas? }\end{array}$ \\
\hline & $\begin{array}{c}\text { Exigencias psicológicas } \\
\text { sensoriales }\end{array}$ & ¿Su trabajo requiere atención constante? \\
\hline \multirow{5}{*}{$\begin{array}{l}\text { Trabajo activo y desarrollo } \\
\text { de habilidades }\end{array}$} & Influencia & $\begin{array}{l}\text { ¿Tiene influencia sobre la cantidad de trabajo que se le } \\
\text { asigna? }\end{array}$ \\
\hline & $\begin{array}{l}\text { Posibilidades de desarrollo en el } \\
\text { trabajo } \\
\end{array}$ & ¿Su trabajo permite que aprenda cosas nuevas? \\
\hline & $\begin{array}{c}\text { Control sobre los tiempos de } \\
\text { trabajo }\end{array}$ & $\begin{array}{l}\text { ¿Puede dejar su trabajo un momento para conversar con un } \\
\text { compañero o compañera? }\end{array}$ \\
\hline & Sentido del trabajo & La tarea que se hace, ¿le parece importante? \\
\hline & Integración en la empresa & $\begin{array}{l}\text { ¿Siente que su empresa tiene una gran importancia para } \\
\text { usted? }\end{array}$ \\
\hline \multirow{5}{*}{$\begin{array}{l}\text { Apoyo social y calidad de } \\
\text { liderazgo }\end{array}$} & Claridad del rol & ¿Sabe exactamente qué tareas son de su responsabilidad? \\
\hline & Conflicto de rol & $\begin{array}{l}\text { ¿Tiene que hacer tareas que usted cree que deberían hacer- } \\
\text { se de otra manera? }\end{array}$ \\
\hline & Calidad de liderazgo & ¿Sus jefes inmediatos resuelven bien los conflictos? \\
\hline & $\begin{array}{l}\text { Calidad de la relación con } \\
\text { los superiores }\end{array}$ & $\begin{array}{l}\text { ¿Recibe ayuda y apoyo de su inmediato o inmediata } \\
\text { superior? }\end{array}$ \\
\hline & $\begin{array}{c}\text { Calidad de la relación con } \\
\text { los compañeros/as de trabajo }\end{array}$ & Entre compañeros y compañeras, ¿se ayudan en el trabajo? \\
\hline \multirow{3}{*}{ Compensaciones } & $\begin{array}{l}\text { Inseguridad respecto del contra- } \\
\text { to de trabajo } \\
\end{array}$ & $\begin{array}{l}\text { ¿Está preocupado por si le despiden o no le renuevan el } \\
\text { contrato? }\end{array}$ \\
\hline & $\begin{array}{l}\text { Inseguridad respecto a las } \\
\text { características del trabajo }\end{array}$ & $\begin{array}{l}\text { ¿Está preocupado por si le cambian de tareas contra su } \\
\text { voluntad? }\end{array}$ \\
\hline & Estima & Mis superiores me dan el reconocimiento que merezco \\
\hline \multirow{2}{*}{ Doble presencia } & Carga de tareas domésticas & $\begin{array}{l}\text { Si está ausente un día de su casa, ¿las tareas domésticas } \\
\text { que realiza se quedan sin hacer? } \\
\end{array}$ \\
\hline & $\begin{array}{c}\text { Preocupación por tareas domés- } \\
\text { ticas }\end{array}$ & $\begin{array}{c}\text { Cuando está en el trabajo, ¿piensa en las exigencias domés- } \\
\text { ticas y familiares? }\end{array}$ \\
\hline
\end{tabular}

Fuente: Acevedo, Sánchez, Farías y Fernández (2013). Riesgos Psicosociales en el Equipo de Salud de Hospitales Públicos de la Provincia de Córdoba, Argentina. 


\section{Análisis estadístico}

Los datos surgidos de las encuestas fueron guardados directamente en una base de datos para su procesamiento y análisis posterior. Para lo anterior se utilizó el programa SPSS 20.0 para windows, en el que se el análisis estadístico descriptivo de los datos con el objetivo de explorar el comportamiento de las dimensiones estudiadas en la muestra bajo análisis. Las respuestas fueron clasificadas según su nivel de riesgo: alto (exposición psicosocial más desfavorable para la salud), medio (exposición psicosocial intermedia) y bajo (exposición psicosocial más favorable para la salud). Para el análisis de resultados se utilizaron estadísticas descriptivas según el tipo de variables; para variables cualitativas, frecuencias absolutas y relativas, y para las variables cuantitativas, medias y desviaciones estándar, y además se aplicó el test Kruskal Wallis $(p<0,05)$.

\section{Resultados}

\section{Características socio-demográficas de los participantes del estudio}

De los 317 trabajadores se encuestó a un total de 298, en el que un 90,9\% correspondió a hombres y el $9,1 \%$ a mujeres. La mayoría de los trabajadores estaba en un rango de edad entre 26 y 35 años $(35,9 \%)$, seguido de 36 y 45 años (29,5\%), luego entre 46 y 55 años $(15,8 \%)$, mayores a 55 años $(10,4 \%)$ y finalmente el menor porcentaje estuvo en los menores de 26 años con un $8,4 \%$ de la muestra. En cuanto a las secciones en las que los trabajadores desempeñan sus funciones, el porcentaje mayor correspondió a PO $(45,3 \%)$, seguido por $\operatorname{OEM}(23,2 \%)$, luego el personal ADMO (12,1\%), LMS $(10,4 \%)$ y finalmente AG $(9,1 \%)$. En relación al nivel de escolaridad, el $35,6 \%$ de los trabajadores posee solamente educación media, un 23,2\% posee educación técnica, un $22,8 \%$ solo educación básica, un $16,8 \%$ tiene un título universitario y un $1,7 \%$ no educación. En la Tabla 2 se exponen los resultados que describen la composición de los trabajadores estudiados.
Tabla 2:

Características socio-demográficas de la muestra.

\begin{tabular}{|c|c|c|c|}
\hline \multicolumn{2}{|c|}{ Datos socio-demográficos } & Frecuencia & $\%$ \\
\hline \multirow[t]{5}{*}{ Edad } & $<25$ & 25 & $8 \%$ \\
\hline & $26-35$ & 107 & $36 \%$ \\
\hline & $36-45$ & 88 & $30 \%$ \\
\hline & $46-55$ & 47 & $16 \%$ \\
\hline & $<56$ & 31 & $10 \%$ \\
\hline \multirow[t]{2}{*}{ Sexo } & Masculino & 271 & $91 \%$ \\
\hline & Femenino & 27 & $9 \%$ \\
\hline \multirow{5}{*}{$\begin{array}{c}\text { Nivel de } \\
\text { escolaridad }\end{array}$} & Sin educación & 5 & $2 \%$ \\
\hline & Educación básica & 68 & $23 \%$ \\
\hline & Enseñanza media & 106 & $36 \%$ \\
\hline & Técnico & 69 & $23 \%$ \\
\hline & Universitario & 50 & $17 \%$ \\
\hline \multirow[t]{2}{*}{ Estado civil } & Casado & 127 & $43 \%$ \\
\hline & Soltero & 171 & $57 \%$ \\
\hline \multirow{4}{*}{$\begin{array}{c}\text { Antigüedad } \\
\text { laboral }\end{array}$} & $1-5$ & 105 & $35 \%$ \\
\hline & $6-10$ & 81 & $27 \%$ \\
\hline & $11-15$ & 77 & $26 \%$ \\
\hline & $>16$ & 35 & $12 \%$ \\
\hline
\end{tabular}

Fuentes: Elaboración propia.

\section{Frecuencia de exposición a riesgos psicosociales en el ámbito portuario}

En la tabla 3 se exponen los resultados que describen las diversas dimensiones por cada grupo. Se encontraron diferencias estadísticamente significativas entre los grupos en las dimensiones exigencias psicológicas ( $p$ value $=0,00)$. Las áreas AG (media $=51,30)$ y $\mathrm{PO}($ media $=$ $52,30)$ se ubican en un rango medio de exposición a este factor y quienes se desempeñan como OEM (media = $56,16), \mathrm{AO}($ media $=58,61)$ y LMS $($ media $=67,74)$ se encuentran en un rango de exposición alto. En cuanto a las sub-dimensiones, los resultados se describen en la tabla 4. Para el caso de la dimensión exigencias psicológicas se encontraron diferencias estadísticamente significativas en cuatro sub-dimensiones: exigencias psicológicas cuantitativas ( $p$ value $=0,031$ ), exigencias psicológicas cognitivas ( $p$ value $=0,00$ ), exigencias psicológicas emocionales ( $p$ value $=0,00$ ) y exigencias psicológicas de esconder emociones ( $p$ value $=0,044$ ). La sub-dimen- 
sión exigencias psicológicas cuantitativas presentó rango de exposición bajo, a excepción del área AG (media = $31,48)$, que obtuvo un rango medio de exposición. A su vez, en la dimensión exigencias psicológicas cognitivas, todas las áreas a excepción de LMS (media = 80,65), obtuvieron rango bajo de exposición.

Tabla 3:

Estadísticos descriptivos y Kruskal Wallis para los factores del cuestionario de evaluación de riesgos psicosociales en el trabajo SUSESO-ISTAS 21, en la variable sección.

\begin{tabular}{|c|c|c|c|c|c|c|c|c|c|c|c|}
\hline $\begin{array}{c}\text { Dimensión de } \\
\text { los factores } \\
\text { psicosociales }\end{array}$ & $\begin{array}{c}\text { Operación de } \\
\text { equipos y } \\
\text { mantenimiento }\end{array}$ & \multicolumn{2}{|c|}{$\begin{array}{c}\text { Línea de mando } \\
\text { y supervisión }\end{array}$} & \multicolumn{2}{|c|}{$\begin{array}{c}\text { Administrativos } \\
\text { operativos }\end{array}$} & \multicolumn{2}{|c|}{$\begin{array}{c}\text { Personal } \\
\text { operativo }\end{array}$} & \multicolumn{2}{|c|}{$\begin{array}{c}\text { Administración y } \\
\text { gerencia }\end{array}$} & \\
\cline { 2 - 13 } & Media & DE & Media & DE & Media & DE & Media & DE & Media & DE & Sig. \\
\hline $\begin{array}{c}\text { Exigencias } \\
\text { psicológicas }\end{array}$ & 56,16 & 17,91 & 67,74 & 15,16 & 58,61 & 14,42 & 52,3 & 14,2 & 51,3 & 13,27 & 0 \\
\hline $\begin{array}{c}\text { Trabajo activo } \\
\text { y desarrollo de } \\
\text { habilidades }\end{array}$ & 28,99 & 16,44 & 23,55 & 13,92 & 33,47 & 12,47 & 30,37 & 14,15 & 30 & 13 & 0,04 \\
\hline $\begin{array}{c}\text { Apoyo social } \\
\text { en la empresa y } \\
\text { calidad de } \\
\text { liderazgo }\end{array}$ & 32,03 & 15,75 & 27,9 & 13,65 & 28,89 & 14,35 & 31 & 13,81 & 30,74 & 15,11 & 0,75 \\
\hline Compensación & 47,46 & 24,1 & 32,26 & 19,57 & 45,83 & 21,87 & 54,2 & 22,37 & 37,35 & 18,69 & 0 \\
\hline Doble presencia & 39,96 & 28,71 & 40,72 & 21,4 & 48,61 & 22,91 & 44,91 & 25,79 & 50,93 & 31,38 & 0,077 \\
\hline
\end{tabular}

Fuente: Elaboración propia.

Para la sub-dimensión exigencias psicológicas emocionales, las áreas AG (media $=48,15)$ y $\mathrm{PO}$ (media $=$ $48,70)$ obtuvieron rangos de exposición bajos. En tanto, las áreas PEM (media $=59,78)$, AO (media $=61,81$ ) y LMS (media $=70,16$ ) obtuvieron rango medio de exposición.

En cuanto a las exigencias psicológicas de esconder emociones, el área de AG (media $=43,52$ ) obtuvo un rango de exposición medio, mientras que todas las otras áreas obtuvieron rangos altos de exposición.

En relación a los resultados de la dimensión trabajo activo y desarrollo de habilidades, se encontraron diferencias significativas al comparar la media de las áreas, por lo tanto se puede observar que los trabajadores que se desempeñan en LMS se encuentran en el rango de exposición a este factor, (media $=23,55)$, mientras que en los resultados de los demás grupos, OEM (media $=28,99)$, AG $($ media $=30)$ PO (media $=$ 30,37 ) y $\mathrm{AO}$ (media $=33,47$ ), se ubican en un rango de exposición medio.
Se dieron diferencias estadísticamente significativas en la sub-dimensión trabajo activo y desarrollo de habilidades, específicamente en control sobre el tiempo de trabajo ( $p$ value $=0,02$ ) e integración en la empresa ( $p$ value $=0,47)$.

La sub-dimensión control sobre el tiempo de trabajo arrojó un rango de exposición medio en LMS (media = 46,77 ). Por otro lado, los grupos AG (media $=52,78$ ), $\mathrm{PO}($ media $=58,33), \mathrm{AO}($ media $=59,03)$ y finalmente OEM (media $=64,49$ ) presentaron rangos medios de exposición.

Para la sección PO, la sub-dimensión integración en la empresa se ubicó en un rango de exposición bajo medio (media $=26,11$ ), mientras que el resto de las secciones, OEM (media $=14,86)$, LMS (media $=16,13$ ), $\mathrm{AO}$ (media $=22,22$ ) y AG (media $=22,22$ ), se encontraron en un rango de exposición bajo.

Para el factor compensación se hallaron diferencias estadísticamente significativas entre las medias para la variable sección ( $p$ value $=0,00$ ). Se ubican en un 
rango medio de exposición a este factor las siguientes secciones: $\mathrm{LMS}$ (media $=32,26$ ) y AG (media $=37,35$ ); mientras que los trabajadores que se desempeñan en la sección AO (media $=45,83)$, OEM $($ media $=47,46)$ y PO (media $=52,20$ ) están en un rango de exposición alto a este factor.

En cuanto a las sub-dimensiones se encontraron diferencias estadísticamente significativas entre las medias de las secciones, y para las sub-dimensiones estima ( $p$ value $=0,02)$ e inseguridad respecto al contrato de trabajo ( $p$ value $=0,00$ )

Para la sub-dimensión estima, LMS se encuentra en un rango de exposición medio (media $=25$ ), y el resto de las secciones logró un rango de exposición alto, obteniéndose las siguientes medias por área: AG (media $=45,37), \mathrm{OEM}($ media $=47,10), \mathrm{AO}($ media $=49,31) \mathrm{y}$ PO $($ media $=56,85)$. En la sub-dimensión inseguridad respecto al contrato de trabajo, todas las secciones se ubican en un rango de exposición medio, obteniéndose las siguientes medias: AG (media $=24,07$ ), LMS (media $=24,19), \mathrm{AO}($ media $=30,56), \mathrm{OEM}($ media $=41,67) \mathrm{y}$ $\mathrm{PO}($ media $=48,33)$.

Por otra parte, las dimensiones apoyo social en la empresa $(p$ value $=0,75)$ y doble presencia $(p$ value $=$ $0,077)$ no presentaron diferencias estadísticamente significativas entre las secciones. Para la dimensión apoyo social en la empresa, las secciones OEM (media $=32,03$ ), $\mathrm{PO}($ media $=31)$, $\mathrm{AG}($ media $=30,74), \mathrm{AO}$ (media $=$ 28,89 ) y LMS (media $=27,9$ ) se encontraron en un rango de exposición medio. En tanto, para la dimensión doble presencia, las secciones PO (media $=44,91$ ), AO (media $=48,61$ ) y AG (media $=50,93$ ) se encuentran en un rango de exposición alto, mientras que las secciones LMS (media $=40,71)$ y OEM (media $=39,96$ ) se encontraron en un rango de exposición medio.

Tabla 4:

Estadísticos descriptivos y Kruskal Wallis para las sub-dimensiones de los factores del cuestionario de evaluación de riesgos psicosociales en el trabajo SUSESO-ISTAS 21, en la variable sección.

\begin{tabular}{|c|c|c|c|c|c|c|c|c|c|c|c|}
\hline \multirow[t]{2}{*}{$\begin{array}{l}\text { Sub-dimensiones de los } \\
\text { factores }\end{array}$} & \multicolumn{2}{|c|}{$\begin{array}{l}\text { Operaciones de } \\
\text { equipos de } \\
\text { mantención }\end{array}$} & \multicolumn{2}{|c|}{$\begin{array}{l}\text { Línea de mando } \\
\text { y supervisión }\end{array}$} & \multicolumn{2}{|c|}{$\begin{array}{c}\text { Administrativo } \\
\text { operativo }\end{array}$} & \multicolumn{2}{|c|}{$\begin{array}{l}\text { Personal } \\
\text { operativo }\end{array}$} & \multicolumn{2}{|c|}{$\begin{array}{l}\text { Administración } \\
\text { y gerencia }\end{array}$} & \multirow[t]{2}{*}{ Sig. } \\
\hline & Media & $\mathrm{DE}$ & Media & $\mathrm{DE}$ & Media & $\mathrm{DE}$ & Media & $\mathrm{DE}$ & Media & $\mathrm{DE}$ & \\
\hline $\begin{array}{c}\text { ¿Puede hacer su trabajo } \\
\text { con tranquilidad y tenerlo } \\
\text { al día? }\end{array}$ & 19,57 & 23,04 & 28,23 & 19,09 & 24,31 & 23,52 & 22,04 & 23,86 & 31,48 & 20,32 & ,031 \\
\hline $\begin{array}{c}\text { En su trabajo, ¿tiene usted } \\
\text { que tomar decisiones } \\
\text { difíciles? }\end{array}$ & 23,24 & 27,34 & 80,65 & 25,58 & 56,25 & 26,98 & 50,19 & 26,89 & 47,22 & 26,25 & , 000 \\
\hline $\begin{array}{l}\text { En general, Considera } \\
\text { usted que su trabajo le } \\
\text { produce desgaste } \\
\text { emocional? }\end{array}$ & 59,78 & 34,36 & 70,16 & 26,94 & 61,81 & 24,99 & 48,70 & 26,68 & 48,15 & 26,79 & ,000 \\
\hline $\begin{array}{l}\text { En su trabajo, ¿tiene usted } \\
\text { que guardar sus emocio- } \\
\text { nes y no expresarlas? }\end{array}$ & 51,81 & 36,46 & 62,90 & 29,49 & 64,58 & 28,27 & 54,44 & 33,58 & 43,52 & 21,48 & , 044 \\
\hline $\begin{array}{l}\text { ¿Su trabajo requiere aten- } \\
\text { ción constante? }\end{array}$ & 91,30 & 21,36 & 96,77 & 10,69 & 86,11 & 26,35 & 86,11 & 23,79 & 86,11 & 23,34 & , 075 \\
\hline
\end{tabular}




\begin{tabular}{|c|c|c|c|c|c|c|c|c|c|c|c|}
\hline \multirow[t]{2}{*}{$\begin{array}{l}\text { Sub-dimensiones de los } \\
\text { factores }\end{array}$} & \multicolumn{2}{|c|}{$\begin{array}{l}\text { Operaciones de } \\
\text { equipos de } \\
\text { mantención }\end{array}$} & \multicolumn{2}{|c|}{$\begin{array}{l}\text { Línea de mando } \\
\text { y supervisión }\end{array}$} & \multicolumn{2}{|c|}{$\begin{array}{c}\text { Administrativo } \\
\text { operativo }\end{array}$} & \multicolumn{2}{|c|}{$\begin{array}{l}\text { Personal } \\
\text { operativo }\end{array}$} & \multicolumn{2}{|c|}{$\begin{array}{c}\text { Administración } \\
\text { y gerencia }\end{array}$} & \multirow[t]{2}{*}{ Sig. } \\
\hline & Media & $\mathrm{DE}$ & Media & $\overline{\mathrm{DE}}$ & Media & $\overline{D E}$ & Media & $\overline{D E}$ & Media & $\mathrm{DE}$ & \\
\hline $\begin{array}{l}\text { ¿Tiene influencia sobre la } \\
\text { cantidad de trabajo que } \\
\text { se le asigna? }\end{array}$ & 36,96 & 38,25 & 29,03 & 26,69 & 47,92 & 33,47 & 37,78 & 31,43 & 36,11 & 24,35 & ,202 \\
\hline $\begin{array}{l}\text { ¿Su trabajo permite que } \\
\text { aprenda cosas nuevas? }\end{array}$ & 64,49 & 29,83 & 46,77 & 16,76 & 59,03 & 21,67 & 58,33 & 23,26 & 52,78 & 16,01 & 2,002 \\
\hline $\begin{array}{l}\text { ¿Puede dejar su trabajo } \\
\text { un momento para con- } \\
\text { versar con un compañero } \\
\text { o compañera? }\end{array}$ & 21,74 & 23,84 & 22,58 & 28,40 & 27,78 & 28,52 & 19,07 & 26,66 & 27,78 & 26,25 & 147 \\
\hline $\begin{array}{c}\text { La tarea que se hace, ¿le } \\
\text { parece importante? }\end{array}$ & 6,88 & 13,48 & 3,23 & 8,52 & 10,42 & 16,23 & 10,56 & 19,43 & 11,11 & 14,43 & ,126 \\
\hline $\begin{array}{l}\text { ¿Siente que su empresa } \\
\text { tiene una gran importan- } \\
\text { cia para usted? }\end{array}$ & 14,86 & 24,75 & 16,13 & 21,94 & 22,22 & 24,48 & 26,11 & 29,75 & 22,22 & 25,32 & ,047 \\
\hline $\begin{array}{c}\text { ¿Sabe exactamente qué } \\
\text { tareas son de su respon- } \\
\text { sabilidad? }\end{array}$ & 10,87 & 16,31 & 8,06 & 13,52 & 15,28 & 22,58 & 11,48 & 19,74 & 13,89 & 17,45 & ,620 \\
\hline $\begin{array}{c}\text { ¿Tiene que hacer tareas } \\
\text { que usted cree que de- } \\
\text { berían hacerse de otra } \\
\text { manera? }\end{array}$ & 48,55 & 20,95 & 54,84 & 23,65 & 51,39 & 22,32 & 51,30 & 21,67 & 47,22 & 28,02 &, 540 \\
\hline $\begin{array}{l}\text { ¿Sus jefes inmediatos } \\
\text { resuelven bien los } \\
\text { conflictos? }\end{array}$ & 39,13 & 32,51 & 25,81 & 18,80 & 29,17 & 23,53 & 35,37 & 24,89 & 32,41 & 26,69 & 243 \\
\hline $\begin{array}{l}\text { ¿Recibe ayuda y apoyo } \\
\text { de su inmediato o inme- } \\
\text { diata superior? }\end{array}$ & 19,57 & 23,82 & 18,55 & 18,22 & 17,36 & 21,40 & 17,22 & 23,55 & 25,93 & 22,45 & 199 \\
\hline $\begin{array}{l}\text { Entre compañeros y com- } \\
\text { pañeras, ¿se ayudan en el } \\
\text { trabajo? }\end{array}$ & 42,03 & 28,28 & 32,26 & 25,16 & 31,25 & 24,18 & 39,63 & 25,44 & 34,26 & 24,17 & 143 \\
\hline $\begin{array}{c}\text { ¿Está preocupado por si } \\
\text { le despiden o no le re- } \\
\text { nuevan el contrato? }\end{array}$ & 47,10 & 39,42 & 25,00 & 31,62 & 49,31 & 38,03 & 56,85 & 40,57 & 45,37 & 35,38 & ,002 \\
\hline $\begin{array}{l}\text { ¿Está preocupado por } \\
\text { si le cambian de tareas } \\
\text { contra su voluntad? }\end{array}$ & 41,67 & 35,27 & 24,19 & 28,49 & 30,56 & 31,69 & 48,33 & 34,04 & 24,07 & 25,46 & ,000 \\
\hline
\end{tabular}




\begin{tabular}{|c|c|c|c|c|c|c|c|c|c|c|c|}
\hline \multirow[t]{2}{*}{$\begin{array}{l}\text { Sub-dimensiones de los } \\
\text { factores }\end{array}$} & \multicolumn{2}{|c|}{$\begin{array}{c}\text { Operaciones de } \\
\text { equipos de } \\
\text { mantención }\end{array}$} & \multicolumn{2}{|c|}{$\begin{array}{l}\text { Línea de mando } \\
\text { y supervisión }\end{array}$} & \multicolumn{2}{|c|}{$\begin{array}{c}\text { Administrativo } \\
\text { operativo }\end{array}$} & \multicolumn{2}{|c|}{$\begin{array}{l}\text { Personal } \\
\text { operativo }\end{array}$} & \multicolumn{2}{|c|}{$\begin{array}{c}\text { Administración } \\
\text { y gerencia }\end{array}$} & \multirow[t]{2}{*}{ Sig. } \\
\hline & Media & $\mathrm{DE}$ & Media & $\mathrm{DE}$ & Media & $\mathrm{DE}$ & Media & $\mathrm{DE}$ & Media & $\mathrm{DE}$ & \\
\hline $\begin{array}{c}\text { Mis superiores me dan } \\
\text { el reconocimiento que } \\
\text { merezco }\end{array}$ & 53,62 & 35,43 & 47,58 & 28,40 & 57,64 & 29,77 & 57,41 & 29,94 & 42,59 & 26,69 & 144 \\
\hline $\begin{array}{c}\text { Si está ausente un día } \\
\text { de su casa, ilas tareas } \\
\text { domésticas que realiza se } \\
\text { quedan sin hacer? }\end{array}$ & 29,35 & 34,82 & 33,87 & 26,26 & 43,06 & 33,06 & 34,07 & 31,54 & 49,07 & 40,12 & 071 \\
\hline $\begin{array}{c}\text { Cuando está en el tra- } \\
\text { bajo, ¿piensa en las } \\
\text { exigencias domésticas y } \\
\text { familiares? }\end{array}$ & 44,57 & 35,32 & 47,58 & 23,59 & 54,17 & 30,76 & 55,74 & 33,45 & 52,78 & 30,49 & 159 \\
\hline
\end{tabular}

\section{DisCUSIÓN}

Los cambios en el mundo del trabajo en las últimas décadas han repercutido en las condiciones de empleo y en los riesgos laborales a los que están expuestos los trabajadores, en particular en el sector portuario, donde las características de las actividades, sumado a nuevos procesos de modernización y cambios, generan condiciones favorables para la aparición de riesgos psicosociales en los trabajadores portuarios (Maciel et al., 2015).

El proceso de modernización del trabajo portuario produce profundas alteraciones en los procesos y en la organización del trabajo, reconfigurando la cultura de los trabajadores portuarios, en sus experiencias de salud, enfermedades y accidentes de trabajo (Machin, Silva y Thereza, 2009). El modo en que el trabajo es ejecutado en situaciones adversas propicia la generación de situaciones de riesgo para la salud física (problemas cardiovasculares, musculares) y psicológicas (asociadas al estrés) de los trabajadores (Bernardo, 2009).

En la presente investigación se describió los resultados en las 5 dimensiones del instrumento, donde exigencias psicológicas, apoyo social en la empresa y calidad de liderazgo, compensaciones y doble presencia se encuentran en un rango alto de exposición, es decir, los trabajadores en estos factores están expuestos a la situación más desfavorable para la salud, mientras que la dimensión trabajo activo y desarrollo de habilidades se encuentra en un rango de exposición bajo.
Con respecto a la comparación entre dimensiones, se hallaron diferencias estadísticamente significativas en el nivel de riesgo de tres dimensiones con respecto a las diferentes secciones de la empresa. Una de estas diferencias se da entre las secciones de la dimensión exigencias psicológicas, donde las áreas OEM, LMS y AO perciben una exposición alta al factor, considerando que estas áreas están directamente asociadas a elementos estresores identificables. Ejemplo de esto es que el personal AP cumple funciones en la atención a clientes, relacionándose de forma inmediata con el público, lo que genera conflicto puesto que éstos determinarán gran parte del servicio entregado por la organización, recayendo responsabilidades y presiones de tono mayor. Las áreas LMS y OEM están supeditadas a un trabajo cargado de exigencias en materia psicológica, debido a las constantes presiones ejercidas por cumplir los compromisos contractuales, y asociadas a un permanente aumento de la productividad y reducción de costos. Lo hallado refuerza lo estipulado en el manual para la evaluación de riesgos psicosociales en el trabajo de la Istas 21 (2002), la cual señala:

Las exigencias psicológicas tienen una doble vertiente: cuantitativa y cualitativa. Desde el punto de vista cuantitativo, las exigencias psicológicas se refieren al volumen de trabajo con relación al tiempo disponible para hacerlo (presión de tiempo) y las interrupciones que obligan a dejar momentáneamente las tareas y volver a ellas más tarde. Desde el punto de vista cualitativo, las exigencias psicológicas se refieren a algunos 
aspectos de la naturaleza de éstas: el trabajo emocional (trabajo que expone a las personas a procesos de transferencia de emociones y sentimientos, como todos aquellos que requieren contacto con usuarios, público y clientes), trabajo cognitivo (que requiere gran esfuerzo intelectual) o trabajo sensorial (esfuerzo de los sentidos). (p. 15).

Por su parte, la dimensión trabajo activo y desarrollo de habilidades obtuvo diferencias estadísticamente significativas entre las secciones. Es así como los trabajadores del área de LMS consideran que no tienen influencia sobre su trabajo ni en la relación con los compañeros; además sienten que su trabajo no es fuente de oportunidades de desarrollo de habilidades y conocimientos, y sienten que no tienen influencia sobre los tiempos de descanso, como vacaciones o detenciones temporales; no experimentan pertenencia sobre el trabajo, es decir, no lo pueden relacionar con otros valores y no se sienten implicados en la empresa. Los trabajadores de las áreas OEM, AO, PO y AG se agrupan en niveles medios, determinando que sus condiciones no son del todo desfavorables, lo que se condice con el área de LMS.

En cuanto a la dimensión de compensación, se pudo observar diferencias significativas entre las secciones, siendo los trabajadores de las áreas AO, OEM y $\mathrm{PO}$ quienes se encuentran en el nivel de riesgo alto, no sintiendo reconocimiento de los superiores por el esfuerzo realizado para desempeñar su trabajo y sintiendo inseguridad con respecto al contrato de trabajo (posibles despidos) e inseguridad en relación a las características mismas del trabajo, cambios de lugar de trabajo, de turnos, etc. (Gil-Monte, 2012).

Acerca de las secciones sin diferencias significativas, apoyo social en la empresa y calidad de liderazgo y doble presencia, se puede concluir lo siguiente: En la dimensión apoyo social en la empresa los trabajadores obtuvieron niveles de riesgo medio en todas las secciones, mientras que en la dimensión doble presencia, tres secciones percibieron riesgo alto al factor, $\mathrm{PO}, \mathrm{AO}$ y AG, lo que guarda estricta relación con el hecho de que en estas secciones se encuentra el mayor número de mujeres trabajadoras del puerto, compartiendo lo descrito por Matosas y Silveira (2003), quienes señalan que las mujeres poseen mayor riesgo o están más expuestas a caer en este tipo de problemática, dadas las condiciones sociales en las que se ven insertas, teniendo que cumplir un rol productivo asociado a un desarrollo laboral y profesional, y un rol familiar vinculado muchas veces a la crianza y responsabilidades en este ámbito. Sin embargo, este factor no se manifiesta solo en mujeres, en vista que los hombres también están expuestos a la doble presencia. Lo descrito se puede ver exacerbado dadas las condiciones, calidad, extensión y características de los nuevos estilos de trabajo.

El estudio de los riesgos psicosociales y de sus correspondientes efectos tiene gran relevancia hoy en día, a medida que se dan a conocer las principales secuelas que la globalización del mercado laboral ha generado en la calidad de vida de los trabajadores (Garrido et al., 2011).

Los trabajadores portuarios han estado en constantes cambios, explicados principalmente por los procesos de modernización, los cuales han generado cambios en las formas de gestión y de organización del trabajo al interior de los terminales portuarios. Estos cambios se han traducido principalmente en una flexibilización de las relaciones laborales y en incremento del control y presión por la productividad, aumentando de esta forma el número de accidentes (Maciel et al., 2015).

Estos cambios han generado un escenario favorable para el aumento de prevalencia de enfermedades mentales y el incremento del nivel de riesgos psicosociales en el rubro portuario, donde se hace necesario la modificación de los espacios y de la organización del trabajo, para poder, desde una perspectiva de la terapia ocupacional, disminuir el alto nivel de riesgos psicosociales percibidos por los trabajadores.

En ciertos estudios se han encontrado correlaciones negativas entre los factores de riesgos psicosociales y satisfacción laboral (Gómez, Hernández y Méndez, 2014), lo que concuerda con otras publicaciones, que señalan que los factores de riesgo psicosociales influyen en la satisfacción laboral en contextos organizacionales en que las condiciones psicosociales, de seguridad y medio ambiente laboral físico implican riesgos para la salud y seguridad de los trabajadores, como sería el caso del personal que desarrolla sus actividades en áreas portuarias (Maciel et al., 2015).

Para futuras investigaciones se sugiere comparar la variable sexo y correlacionar las dimensiones y/o subdimensiones con otros indicadores de salud ocupacional, como la salud mental y otros indicadores de seguridad laboral como la tasa de accidentabilidad u otros.

Se recomienda utilizar metodología mixta, agregando información de tipo cualitativo, para poder articular ambas perspectivas, a fin de lograr análisis más profundos y detallados de los datos levantados. Resultaría interesante comparar los resultados con otras empresas del mismo rubro e indagar, por ejemplo, en similitudes y diferencias en los riesgos percibidos y sus potenciales causas. 
Es necesario orientar acciones efectivas de promoción de ambientes de trabajo más saludables y de prevención de estos riesgos, en particular los vinculados a las dimensiones de exigencias psicológicas, trabajo activo y desarrollo de habilidades y compensación, que fueron aquellas donde se encontraron diferencias significativas entre las secciones.

\section{REFERENCIAS BIBLIOGRÁFICAS}

Acevedo, G., Sánchez, J., María, A. y Fernández, A. (2013). Riesgos Psicosociales en el Equipo de Salud de Hospitales Públicos de la Provincia de Córdoba, Argentina. Ciencia \& trabajo, 15(48), 140-147. https://dx.doi.org/10.4067/S0718-24492013000300006.

Ala-Mursula, L., Vahtera, J., Linna, A., Pentti, J. \& Kivimäki, M. (2005). Employee worktime control moderates the effects of job strain and effort-reward imbalance on sickness absence: the 10-town study. Journal of Epidemiology and Community Health, 59(10), 851-857.

Alvarado, R., Pérez-Franco, J., Saavedra, N., Fuentealba, C., Alarcón, A., Marchetti, N. y Aranda, W. (2012). Validación de un cuestionario para evaluar riesgos psicosociales en el ambiente laboral en Chile. Revista Médica de Chile, 140(9), 1154-1163.

Antunes, R. \& Alves, G. (2004). As mutações no mundo do trabalho na era da mundialização do capital. Educação e Sociedade, 25(87), 335-351.

Asociación Chilena de Seguridad (2016). Procedimiento del Programa de vigilancia de riesgos psicosociales para empresas. Chile. Enero: 35.

Astudillo, P. (2014). Factores psicosociales en el trabajo: ¿Cómo comprender las tensiones psicosociales para regularlas?. Instituto de Salud Pública de Chile, Departamento de Salud Ocupacional. Nota técnica (004-2014).

Barattini, M. (2009). El trabajo precario en la era de la globalización: ¿Es posible la organización? Polis (Santiago), 8(24), 17-37.

Beltrán, C. A., Gónzalez, J. L. L. y Salas, J. H. B. (2013). Factores psicosociales y síndrome de burnout en trabajadores de la industria de la transformación de la masa. Tepic, México. Revista Colombiana de Psiquiatría, 42(2), 167-172.

Bernardo, M. H. (2009). Trabalho duro, discurso flexível: uma análise das contradições do toyotismo a partir da vivência de trabalhadores. Editora Expressão Popular.

Bourdieu P. (2000). "Cortafuegos: reflexiones para servir a la resistencia contra la invasión neoliberal". Anagrama. Barcelona.

Ceballos Vásquez, P., Valenzuela Suazo, S. y Paravic Klijn, T. (2014). Factores de riesgos psicosociales en el trabajo: género y enfermería. Avances en Enfermería, 32(2), 271-279.

Costa, J. A. D. (2010). Mais trabalho! A intensificação do labor na sociedade contemporânea. Sociedade e Estado, 25(2), 371-375.
Cox, T., Griffiths, A. \& Rial-González, E. (2000). Research on workrelated stress: European Agency for Safety and Health at Work. Luxembourg: Office for Official Publications of the European Communities.

Cuenca, R. (1996). Introducción a los riesgos laborales de naturaleza psicosocial. Madrid: Instituto Nacional de Seguridad e Higiene en el Trabajo.

Dormann, C. \& Zapf, D. (2004). Customer-related social stressors and burnout. Journal of occupational health psychology, 9(1), 61.

Feo Istúriz, O. (2003). Reflexiones sobre la globalización y su impacto sobre la salud de los trabajadores y el ambiente. Ciência \& Saúde Coletiva, 8(4), 887-896.

Ferreira, M., Lara, R. \& Mariano, A. (2015). "Trabalho e Saúde dos Estivadores do Porto de Lisboa". International Journal on Working Conditions. Portugal: 10, 120-137.

Garrido Pinzón, J., Blanch Ribas, J. M., Uribe-Rodríguez, A. F., Flórez Acevedo, J. y Pedrozo Sánchez, M. C. (2011). El Capitalismo organizacional como factor de riesgo psicosocial:: Efectos psicológicos colaterales de las nuevas condiciones de trabajo en hospitales y universidades de naturaleza pública. Resultados Santander, Colombia. Psicología desde el Caribe, (28), 166-196.

Gil-Monte, P. R. (2012). Riesgos psicosociales en el trabajo y salud ocupacional. Revista peruana de Medicina Experimental y Salud pública, 29(2), 237-241.

Gómez Rojas, P., Hernández Guerrero, J. y Méndez Campos, M. D. (2014). Factores de riesgo psicosocial y satisfacción laboral en una empresa chilena del área de la minería. Ciencia \& trabajo, 16(49), 9-16

Grau, A., Flichtentrei, D., Suñer, R., Prats, M. y Braga, F. (2009). El síndrome de burnout en profesionales sanitarios hispanoamericanos y españoles. Influencia de factores personales, profesionales y nacionales. Rev Esp Salud Pública, 8, 215-230.

Instituto Sindical de Trabajo, Ambiente y Salud (CopSOQ). (2002). "Manual para la evaluación de riesgos psicosociales en el trabajo". Barcelona, España.

Machin, R., Couto, M. T. \& Rossi, C. C. S. (2009). Representações de trabalhadores portuários de Santos-SP sobre a relação trabalhosaúde. Saúde e Sociedade. 18(4), 639-651.

Maciel, R. H., Gonçalves, R. C., Matos, T. G. R., Fontenelle, M. F. \& Santos, J. B. F. D. (2015). Análise do trabalho portuário: transformações decorrentes da modernização dos portos. Revista Psicologia Organizações e Trabalho, 15(3), 309-321.

Matosas, A. y Silveira, S. (2003). Género y economía informal en América Latina. Nuevos retos y respuestas posibles desde las políticas de formación del trabajo. Boletín Cinterfor: Boletín Técnico Interamericano de Formación Profesional, (155), 231-262.

Ministerio de Salud de Chile (2013). Protocolo de vigilancia de riesgos psicosociales en el trabajo. Departamento de Salud Pública.

OIT-OMS, C. M. (1984). Factores psicosociales en el trabajo: Naturaleza, incidencia y prevención. Medicina del Trabajo. $9^{a}$ Reunión. 
Silla, J. M. P. y Molina, I. R. (2008). Estrés laboral, liderazgo y salud organizacional. Papeles del psicólogo, 29(1), 68-82.

Siegrist, J. (1996). Adverse health effects of high-effort/low-reward conditions. Journal of occupational health psychology, 1(1), 27.

Stoetzer, U., Ahlberg, G., Johansson, G., Bergman, P., Hallsten, L., Forsell, Y. \& Lundberg, I. (2009). Problematic interpersonal relationships at work and depression: a Swedish prospective cohort study. Journal of occupational health, 51(2), 144-151.

Superintendencia de Seguridad Social (2009). SUSESO-ISTAS 21. Cuestionario de evaluación de riesgos psicosociales en el trabajo. Manual de uso. Ministerio del Trabajo de Chile.

Superintendencia de Seguridad Social (2016). Manual del método del Cuestionario SUSESO-ISTAS 21, versión completa y breve. Ministerio del Trabajo de Chile.

Peiró, J. M. (2004). El sistema de trabajo y sus implicaciones para la prevención de los riesgos psicosociales en el trabajo. Universitas psychologica, 3(2), 179-186.

Teiger, C. (1992, november). "Le travail, cet obscur objet de l'ergonomie. In Actes du Colloque interdisciplinaire". Travail: recherches et prospectives Lyon. 30, 111-126.

Torres, A. (2007). "A Lei de Execução Penal e as Atribuições do Serviço Social no Sistema Penitenciário: Conservadorismo pela via da 'Desassistência' Social'. Crítica à execução penal. Rio de Janeiro, 197-204.

Useche, M. C. (2016). La organización del trabajo en el marco de la globalización. Gaceta Laboral, enero-abril, 67-78.

Vanroelen, C., Levecque, K., Moors, G., Gadeyne, S. \& Louckx, F. (2009). "The structuring of occupational stressors in a Post-Fordist work environment. Moving beyond traditional accounts of demand, control and support". Social science \& medicine, 68(6), 1082-1090. 